\title{
Perfis temáticos dos trabalhos de conclusão de curso de graduação em Biomedicina da Bahiana, Brasil
}

\author{
Thematic profiles of the undergraduate thesis of \\ Biomedicine course of Bahiana, Brazil
}

\section{Lara São Pedro Caldas', Ysis Gabrielle Domingos Santos², Artur Gomes Dias-Lima³, Vanessa Silva Brito ${ }^{4}$, Geraldo Argolo Ferraro5, Gabriel Andrade Nonato Queiroz ${ }^{6}$}

\begin{abstract}
'Escola Bahiana de Medicina e Saúde Pública. Salvador, Bahia, Brasil. ORCID: 0000-0002-7413-818X. laracaldas15.2@bahiana.edu.br 2Escola Bahiana de Medicina e Saúde Pública. Salvador, Bahia, Brasil. ORCID: 0000-0002-1293-6012. ysissantos15.2@bahiana.edu.br

${ }^{3}$ Escola Bahiana de Medicina e Saúde Pública. Salvador, Bahia, Brasil. ORCID: 0000-0002-1656-9598. agdlima1@bahiana.edu.br 4Escola Bahiana de Medicina e Saúde Pública. Salvador, Bahia, Brasil. ORCID: 0000-0001-6648-3904. vanessabrito@bahiana.edu.br ${ }^{5}$ Escola Bahiana de Medicina e Saúde Pública. Salvador, Bahia, Brasil. ORCID: 0000-0003-0563-3184. geraldoferraro@bahiana.edu.br ${ }^{6}$ Autor para correspondência. Escola Bahiana de Medicina e Saúde Pública. Salvador, Bahia, Brasil. ORCID 0000-0003-4453-3709. gabrielqueiroz@bahiana.edu.br
\end{abstract}

RESUMO I INTRODUÇão: O curso de Biomedicina da Escola Bahiana de Medicina e Saúde Pública (EBMSP) foi autorizado pelo Ministério da Educação e Cultura (MEC) em 26 de dezembro de 2002 e reconhecido em 23 de janeiro de 2007, todo o funcionamento é integral, sendo as 4.158 horas de carga horária total do curso divididas entre 8 semestres. $O$ trabalho de conclusão de curso (TCC) é obrigatório para que haja a colação de grau. OBJETIVO: Avaliar os perfis temáticos dos TCCs realizados por estudantes do curso de Biomedicina da EBMSP. MATERIAIS E MÉTODOS: Os TCCs de 2006 a 2018 foram analisados através dos resumos e em seguida foram classificados de acordo com o tipo de estudo (revisão ou experimental), ao modelo (artigo ou monografia), em relação as disciplinas da grade curricular, em relação a áreas da CAPES e em relação a habilitação do biomédico. A tabulação dos dados foi realizada pelo Excel e gráficos foram feitos no Graphpad prism. Foram encontrados um maior número de revisões e monografias. RESULTADOS: A disciplina do curso de Biomedicina mais contemplada nos TCCs foi a microbiologia com 55 trabalhos, (14,21\%), seguida de Parasitologia com 35 (9,04\%) e Imunologia com 33 (8,53\%). Quanto a grande área, Ciências Biológicas foi a habilitação mais frequente e área de atuação a mais contemplada também foi microbiologia. Além disso, o número de TCCs recuperados foi representativo, a maior parte deles foi no formato de revisão de literatura e em estilo de monografia. CONCLUSÃo: Percebeu-se que o armazenamento adequado dos trabalhos é de extrema importância para diversas análises, como atualizações na grade curricular, percepções das disciplinas em relação ao mercado de trabalho, novas habilitações contemporâneas e áreas de pesquisa.
ABSTRACT | BACKGROUND: The Biomedicine course of the Bahian School of Medicine and Public Health (EBMSP) was authorized by the Ministry of Education and Culture (MEC) on December 26, 2002 and recognized on January 23, 2007, the entire operation is the 4,158 hours of total course workload divided by 8 semesters. Undergraduate thesis (UT) is required for degree placement. AIM: To evaluate the thematic profiles of the undergraduate thesis carried out by students of the Biomedicine course of the EBMSP. MATERIALS AND METHODS: UTs from 2006 to 2018 were evaluated through the abstracts and then classified according to the type of study (revision or experimental), style (article or monograph), in relation to the graduation matters, in relation to areas of CAPES and in relation to the areas of biomedicine. The data tabulation was performed by Excel and graphs were done in Graphpad prism. A greater number of reviews and monographs were found. RESULTS: The most frequent discipline of the Biomedicine course was microbiology with 55 papers (14.21\%), followed by Parasitology with 35 (9.04\%) and Immunology with 33 (8.53\%). For the major área of the CAPES, Biological Sciences was the most frequent and the area most frequently occupation area was also microbiology. In addition, the number of retrieved TCCS was representative, most of them in literature review format and monograph style. CONCLUSION: It is noticed that the adequate storage of the works is of extreme importance for several analyzes, such as updates in the curriculum, perceptions of the disciplines in relation to the labor market and research areas.

KEYWORDS: Biomedicine. TCC. Article. Monography.

PALAVRAS-CHAVE: Biomedicina. TCC. Artigo. Monografia. 


\section{Introdução}

A Biomedicina surgiu no ano de 1966 na Escola Paulista de Medicina, sua regulamentação veio em 3 de setembro de 1979, com a Lei $n^{\circ}$ 6.684; seguida da Lei $n^{\circ}$ 6.686, de 11 de setembro de 1979, que deu aos Biomédicos o direito do exercício das análises clínico-laboratoriais. Sendo criados em 30 de agosto de 1982, através da Lei $n^{\circ}$ 7.017, o Conselho Federal (CFBM) e Regionais de Biomedicina (CRBM). No entanto, apenas em 24 de junho de 1986 através da Resolução $n^{\circ} 86$ que definitivamente foi confirmado o direito do Biomédico de exercer as análises clínico-laboratoriais, passando a ser fiscalizado pelo CFBM e CRBM, que fazem a regulamentação e fiscalização como um todo do exercício da profissão!

O curso de Biomedicina da Escola Bahiana de Medicina e Saúde Pública (EBMSP) foi autorizado pelo Ministério da Educação e Cultura (MEC) de acordo com a Portaria 3.883, de 26 de dezembro de 2002 e reconhecido em 23 de janeiro de 2007, pela portaria $\mathrm{n}^{\circ} 68$. O regime de funcionamento é integral, sendo as 4.158 horas de carga horária total do curso divididas entre 8 semestres. Nos 4 anos de curso na EBMSP, o aluno necessita do cumprimento de exigências normativas e regulamentares que os torna conscientes e conectados com o papel do Biomédico no mercado de trabalho².

O trabalho de conclusão de curso (TCC) é pré-requisito para a conclusão do curso e atualmente deve ser apresentado em forma de artigo científico no curso de Biomedicina da EBMSP. A sua produção engloba todos os estudos efetuados no curso de graduação, sendo realizado ao final do curso por todos os discentes sob o auxílio de um orientador. O trabalho segue um cronograma de atividades estabelecidas até a sua apresentação ao corpo docente examinador ${ }^{3}$.

Os TCCs de Biomedicina da EBMSP, de 2006.2 a 2016.1 tiveram a produção opcional para artigo científico ou dissertação monográfica, o que foi modificado no semestre 2017.1, com a obrigatoriedade da entrega do trabalho em formato de artigo científico. O tema para realização do trabalho é escolhido livremente entre orientador e graduando. A defesa é realizada através de apresentação oral, na presença de banca examinadora, a qual é composta pelo orientador e dois convidados (docentes da EBMSP ou de outra instituição de ensino superior) especialistas no tema em questão².

O entendimento das motivações na escolha dos temas para o TCC entre os graduandos e orientadores pode ser útil para correlacionar com as disciplinas do curso de graduação, com as habilitações escolhidas pelos graduandos. Ainda, por meio de pesquisas bem fundamentadas, é possível investigar a importância do trabalho no perfil profissional do graduado, bem como traçar novos perfis da pesquisa científica e subsidiar reflexões internas para o curso de Biomedicina. Desta forma, o presente trabalho teve como objetivo avaliar os perfis temáticos dos TCCs, realizados pelos graduandos do curso de Biomedicina da EBMSP.

\section{Materiais e métodos}

\section{Desenho do estudo}

Trata-se de um estudo retrospectivo, longitudinal, exploratório e quantitativo. Para avaliar os perfis temáticos dos TCCs realizados por estudantes do curso de Biomedicina da EBMSP, foram utilizados os dados dos resumos dos TCCs disponíveis do curso de Biomedicina da EBMSP.

A coleta dos resumos foi realizada através do portal institucional de trabalhos científicos da EBMSP4, os quais disponibilizaram os resumos dos trabalhos de conclusão de curso (TCCs) produzidos no final da graduação de Biomedicina. Todos os resumos foram lidos e classificados por pelo menos dois autores.

\section{Número de formados versus número de TCCs}

A análise inicial foi realizada pela comparação do número absoluto de alunos concluintes do curso de Biomedicina desde a sua primeira turma formada (2006.1) até o último semestre concluído (2018.2). Foram excluídos os anos em que não foi disponibilizado nenhum resumo para avaliação. O número absoluto de estudantes concluintes foi disponibilizado pela secretaria acadêmica da Escola Bahiana de Medicina e Saúde Pública (EBMSP) da Unidade Acadêmica do Cabula. Os dados foram expressos em números absolutos e porcentagem. 
Número de trabalhos experimentais versus revisões de literatura

Aqueles resumos que tratavam de estudos com experimentos animais, experimentos com células, entrevistas, relatos de caso, ecológicos, documentais e demais trabalhos com análises de dados foram classificados como experimentais. Os outros trabalhos foram classificados como revisões da literatura.

\section{Número de monografias versus número de artigos}

Os trabalhos também foram classificados de acordo com o estilo, podendo ser classificados como monografia ou artigo. Até o ano de 2016 o modelo de artigo era opcional. Por este motivo, até 2016 , apenas os TCCs que descreveram no resumo tratar-se de artigo científico foram classificados como tal. A partir de 2017 o modelo de artigo tornou-se obrigatório para todos os trabalhos de conclusão de curso da EBMSP.

\section{Avaliação temática dos TCCs em relação as disciplinas da graduação}

Os resumos foram classificados de acordo com a disciplina do curso de graduação em Biomedicina da EBMSP (Matriz 2017.1-atual)5.

\section{Avaliação temática dos TCCs em relação as áreas da CAPES}

Os resumos também foram classificados de acordo com as áreas do conhecimento da Coordenação de Aperfeiçoamento de Pessoal de Nível Superior CAPES ${ }^{6}$.

As áreas do conhecimento da CAPES têm o objetivo prático de auxiliar as instituições de ensino, pesquisa e inovação, através da sistematização e de temas associados a projetos de pesquisa da área de ciência e tecnologia. A classificação das áreas do conhecimento apresenta 4 níveis, do mais geral ao mais específico ${ }^{7}$.
Quadro 1. Divisão das áreas de conhecimento da CAPES

\begin{tabular}{|c|l|}
\hline $1^{\circ}$ nível & Grande Área \\
\hline $2^{\circ}$ nível & Área do Conhecimento (Área Básica) \\
\hline $3^{\circ}$ nível & Subárea \\
\hline $4^{\circ}$ nível & Especialidade \\
\hline
\end{tabular}

\section{Avaliação temática dos TCCs em relação a habilitação do biomédico}

Os resumos foram adicionalmente classificados de acordo com as habilitações do Biomédico, disponibilizadas pelo Conselho Federal de Biomedicina e pelos CRBMs8. Desde o dia 01 de maio de 2019, Conselho Federal de Biomedicina (CFBM) unificou a lista de habilitações da Biomedicina, unindo algumas áreas sob a denominação "Docência e Pesquisa". Algumas áreas, que antes eram consideradas uma habilitação de forma isolada, agora estão dentro da Docência e Pesquisa, como (Biofísica, virologia, fisiologia, histologia humana, patologia, embriologia, psicobiologia) ${ }^{9}$.

\section{Análise e tabulação dos dados}

O armazenamento e análise dos dados foram realizados através do software Microsoft Office Excel (Microsoft Corporation., Redmond, Washington, EUA). As figuras e análises estatísticas foram feitas através do software Graphpad prism, versão 5.0. Os números foram expressos em número absoluto $(\mathrm{N})$, frequência/porcentagem (\%) média (GraphPad Inc., San Diego, CA, EUA).

\section{Resultados}

\section{Número de formados versus número de TCCs}

Somando-se os anos de 2006 a 2018, 638 estudantes concluíram o curso de Biomedicina da Escola Bahiana de Medicina e Saúde Pública, com uma média de 48,9 estudantes por ano (Figura 1). 


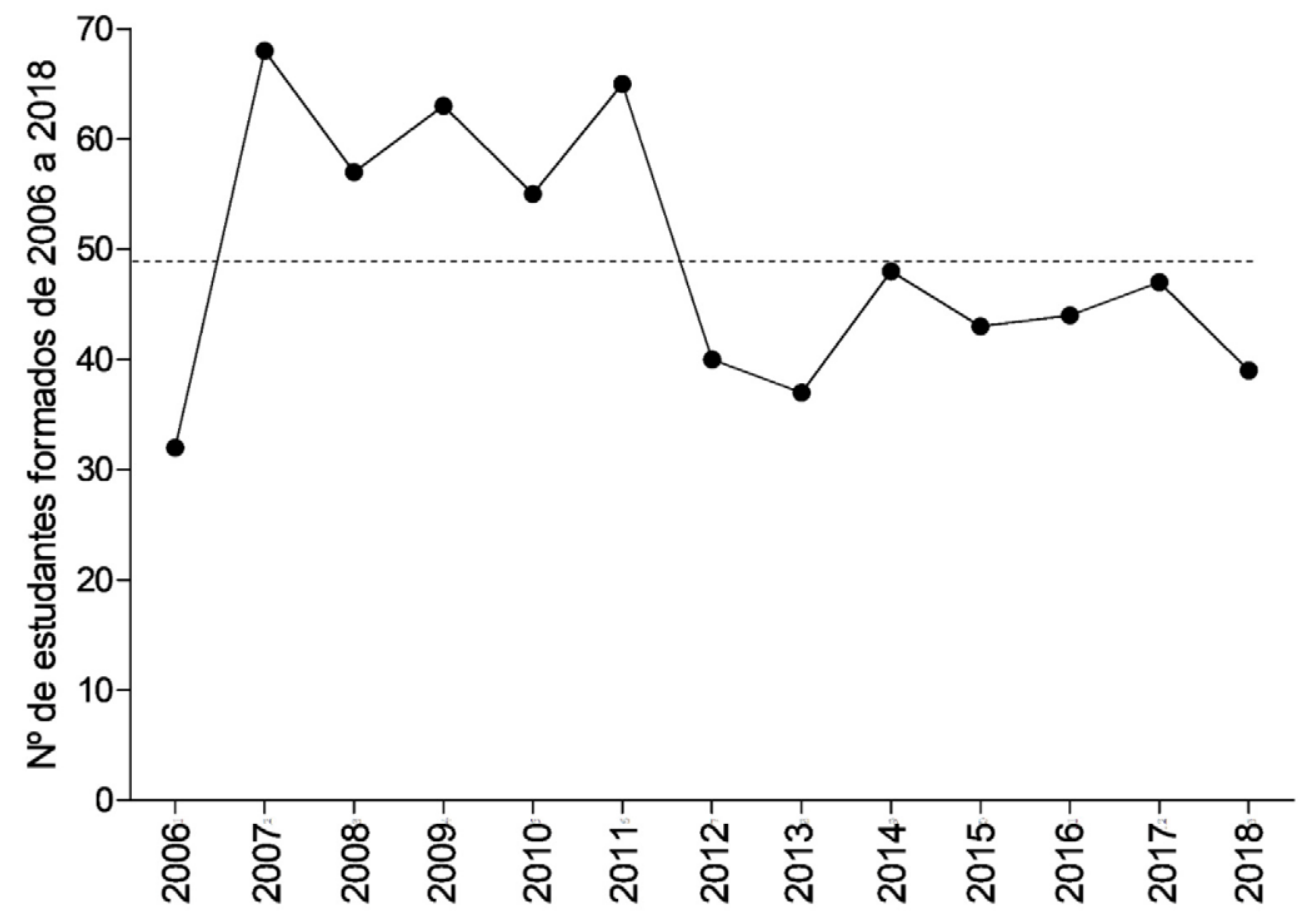

A frequência do $\mathrm{N}^{\circ}$ de concluintes em relação aos TCCs incluídos no presente estudo é observada na figura 2 [Porcentagem do total ( $\mathrm{N}^{\circ}$ de TCCs incluídos / Total de Formados). No ano de 2008 foram incluídos [47,40\% (27/57)] dos TCCs, em 2009 [82,54\% (52/53)], em 2010 [38,18\% (21/55), em 2011 [73,85\% (48/65)], em 2012 [17,50\% (7/40)], em 2013 [81,10\% (30/37)], em 2014 [93,75\% (45/48)], em 2015 [88,37\% (38/43)], em 2016 [95,45\% (42/44)], em 2017 [85,10\% (40/47)], e em 2018 [94,87\% (37/39)]. Desta forma, totalizaram-se 538 formandos dos anos de 2008 a 2018 e foram classificados 387 resumos, correspondendo a 71,93\% (Figura 3). No ano de 2006 e 2007, houve 32 e 68 estudantes concluintes, respectivamente, mas os resumos dos TCCs destes anos não foram encontrados e os dados destes anos foram excluídos do presente trabalho. 
Figura 2. Representação do número de formados versus número de TCCs disponíveis e incluídos no estudo por ano. Os dados são expressos em números absolutos, as barras cinzas a esquerda de cada ano, simboliza o número de formados, a barra branca direita de cada ano simboliza o número de trabalhos disponibilizados

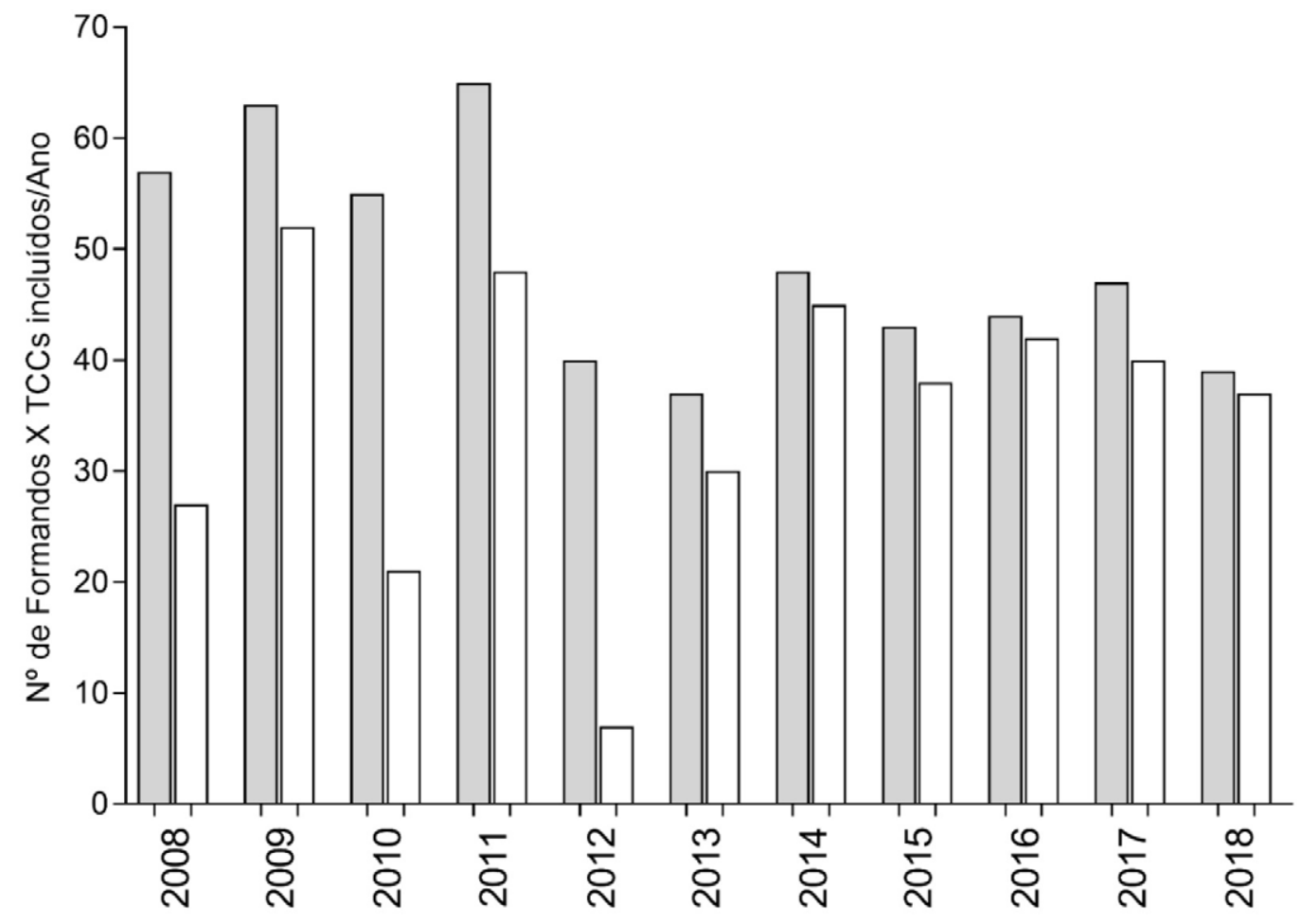

Figura 3. Representação do número absoluto de formados X o número de TCCs disponíveis incluídos no estudo. Os dados são expressos em números absolutos, a barra cinza a esquerda, simboliza o número de formados, a barra branca direita simboliza o número de trabalhos disponibilizados

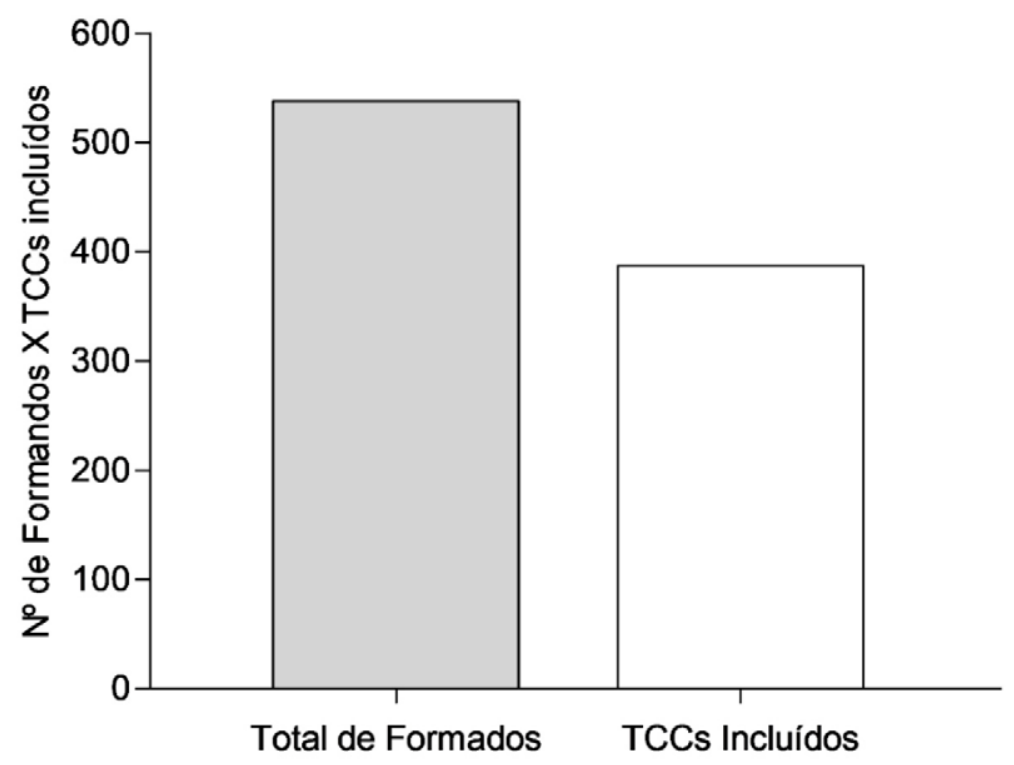


A frequência do número total de estudos no formato de revisão $x$ estudos experimentais são observados na figura 4. Do total de 387 TCCs, [54,26\% (210)] foram no formato de revisão e [45,74\% (177)] foram no formato experimental. A estratificação da frequência do número de estudos no formato de revisão $x$ estudos experimentais por ano são observados na figura 5 . Em relação ao número de TCCs de TCCs de revisão x experimentais por ano, respectivamente, observa-se em 2008 [33,33\% (9) x 66,66\% (18)], em 2009 [30,76\% (16) x 69,23\% (36)], em 2010 [52,38\% (11) x 47,62\% (10)], em 2011 [54,17\% (26) x 45,83\% (22)], em 2012 [71,42\% (5) x 28,58\% (2)], em 2013 [80,00\% (24) x 20,00\% (6)], em 2014 [62,22\% (28) x 37,78\% (17)], em 2015 [78,95\% (24) x 21,05\% (14)], em 2016 [71,43\% (30) x 28,57\% (12)], em 2017 [42,50\% (17) x 57,50\% (23), em 2018 [54,05\% (20) x 45,94\% (17)].

Figura 4. Representação do número absoluto de TCCs no formato de Revisão versus experimentais disponíveis incluídos no estudo. A linha tracejada simboliza o número total absoluto de TCCS

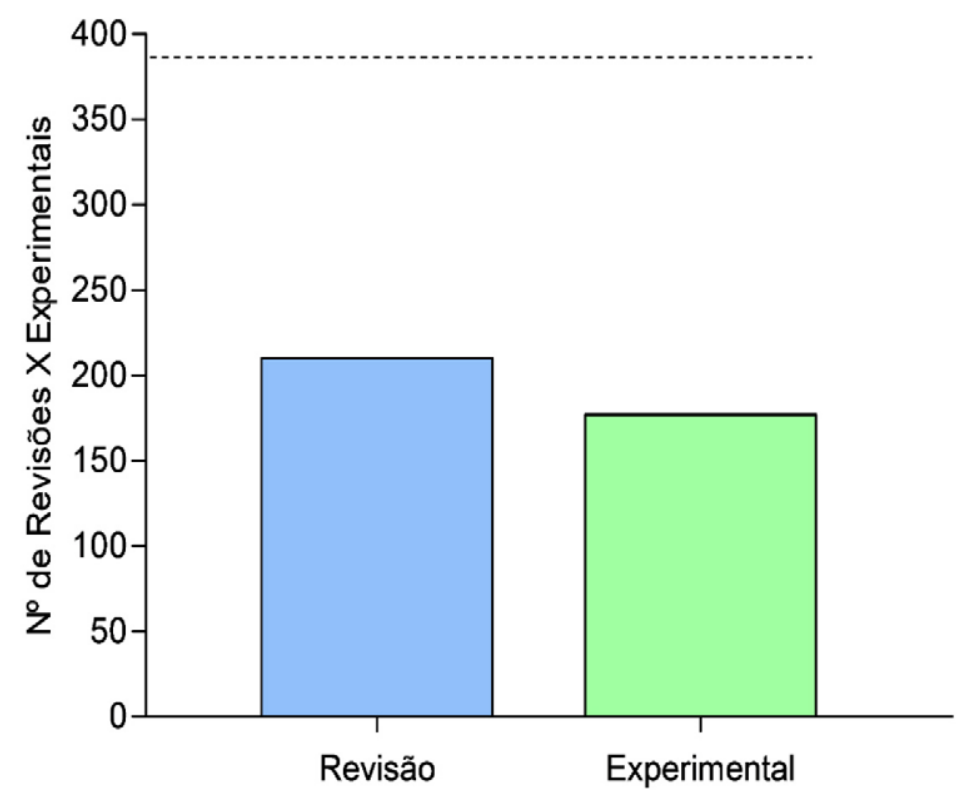


Figura 5. Representação do número absoluto de TCCs no formato de Revisão versus experimentais por anos disponíveis incluídos no estudo. Os dados são expressos em números absolutos, as colunas azuis a esquerda de cada ano, simboliza o número de revisões, as colunas verdes a direita de cada ano simboliza o número de estudos experimentais

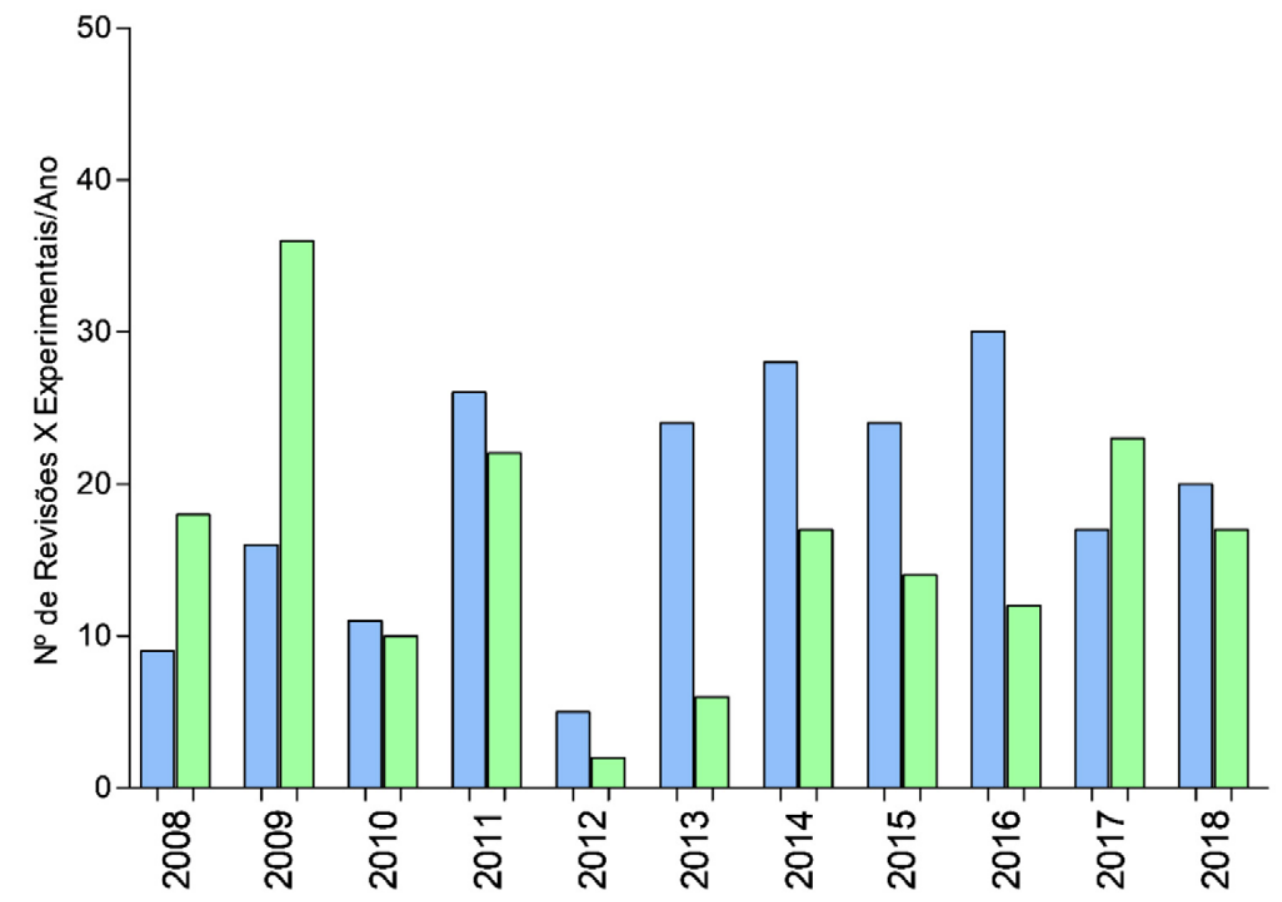

\section{Número de monografias versus número de artigos}

Até o ano de 2016 o modelo de artigo era opcional. Por este motivo, até 2016, apenas os TCCs que descreveram no resumo tratar-se de artigo científico foram classificados como tal, totalizando-se 3 artigos. Já em relação ao número de monografias até 2016, foram contabilizadas 307. A partir de 2017 o modelo de artigo tornou-se obrigatório nos trabalhos de conclusão de curso da EBMSP, totalizando-se 100\% dos TCCs até 2018. Os dados em relação ao modelo de TCC como monografia ou artigo de 2008 a 2018 são observados na figura 6 .

Figura 6. Representação do número absoluto de TCCs no formato de Monografia versus Artigo disponíveis incluídos no estudo. A linha tracejada simboliza o número total absoluto de TCCS

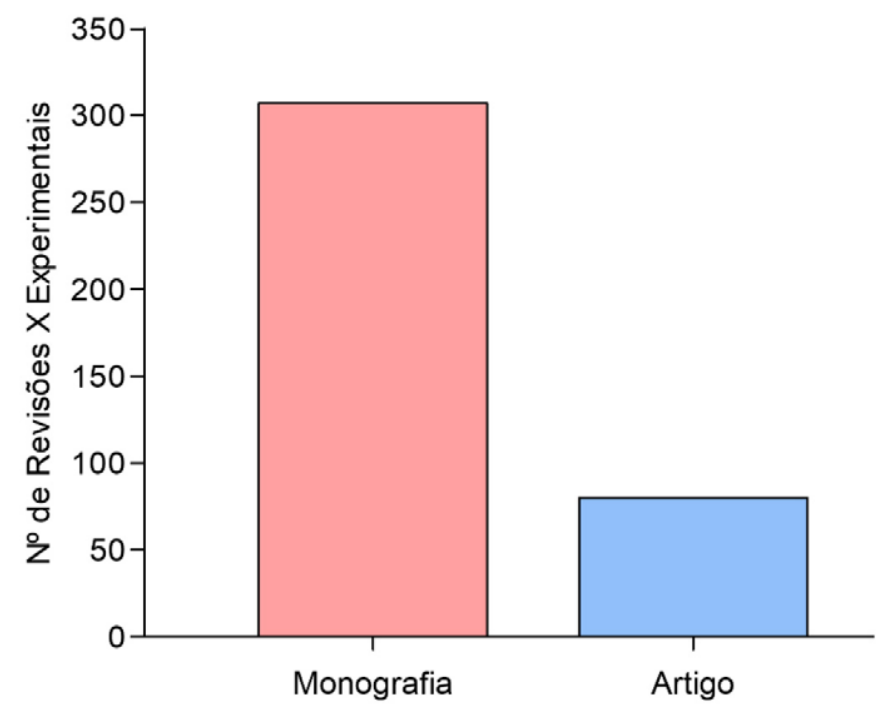




\section{Avaliação temática dos TCCs em relação as disciplinas da graduação}

A disciplina que obteve maior número absoluto de TCCs realizados foi a de Microbiologia, com 55 trabalhos, correspondendo a 14,21\% dos trabalhos, seguida da Parasitologia, com 35 trabalhos, correspondendo a 9,04\%. Além disso, somente os temas das disciplinas de microbiologia e genética [5,4\% (23)] estiveram presentes em trabalhos de todos os anos avaliados (dados não mostrados). Além destas, as disciplinas de Farmacologia [6,72\% (26)] e Imunologia [8,53\% (33)], estiveram presentes em trabalhos de todos os anos avaliados, com exceção dos anos de 2012 e 2013, respectivamente (dados não mostrados). Apenas as disciplinas de Física e Biofísica e Estágio, obtiveram apenas 1 trabalho ao longo dos anos [0,26\% (1)] (Tabela 1).

As disciplinas de Ética e Bioética, Metodologia Instrumental, Primeiros Socorros, Exercício Profissional Biomédico, Anatomia Topográfica, Bioestatística, Seminário Interdisciplinar I e II, Análise instrumental, Avaliação Analítica Integrada e Gestão de Serviços de Saúde e Empreendedorismo não tiveram TCCs relacionados.

Tabela 1. Classificação dos TCCS de acordo com disciplinas do curso de Biomedicina da EBMSP

\begin{tabular}{|c|c|c|c|c|c|c|}
\hline Disciplina & $\begin{array}{c}\text { Semestre } \\
1^{\circ} \text { ao } 8^{\circ}\end{array}$ & $\begin{array}{c}\text { C.H Teórica } \\
\text { Horas }\end{array}$ & $\begin{array}{c}\text { C.H Prática } \\
\text { Horas }\end{array}$ & $\begin{array}{l}\text { C.H Total } \\
\text { Horas }\end{array}$ & $\begin{array}{c}N^{\circ} \text { TCCS } \\
N=387\end{array}$ & $\begin{array}{c}\text { Freq. TCCs } \\
\%\end{array}$ \\
\hline Análises citopatológicas & $6^{\circ}$ & 36 & 18 & 54 & 12 & 3,10 \\
\hline Avaliação ambiental e de produtos & $5^{\circ}$ & 36 & 54 & 90 & 8 & 2,06 \\
\hline Bases de terapias complementares & $5^{\circ}$ & 36 & 18 & 54 & 13 & 3,35 \\
\hline Biologia molecular e celular & $1^{\circ}$ & 54 & 14 & 68 & 7 & 1,80 \\
\hline Biomorfofuncional (1/2) & $1^{\circ} / 2^{\circ}$ & $108 / 108$ & $72 / 72$ & $180 / 180$ & 3 & 0,77 \\
\hline Bioquímica $(1 / 2 / 3)$ & $1 \% / 3^{\circ} / 6^{\circ}$ & $36 / 54 / 36$ & $18 / 0 / 36$ & $54 / 54 / 72$ & 13 & 3,35 \\
\hline Biotecnologia Aplicada & $4^{\circ}$ & 36 & 18 & 56 & 3 & 0,77 \\
\hline Ecologia e Saúde & $3^{\circ}$ & 36 & 36 & 72 & 5 & 1,29 \\
\hline Embriologia e Reprodução assistida & $4^{\circ}$ & 36 & 18 & 54 & 2 & 0,52 \\
\hline Estágio (1/2/3) & $4^{\circ} / 7^{\circ} / 8^{\circ}$ & - & - & $54 / 280 / 550$ & 1 & 0,26 \\
\hline Farmacologia & $6^{\circ}$ & 36 & 18 & 54 & 26 & 6,72 \\
\hline Física e Biofísica & $2^{\circ}$ & 36 & 18 & 54 & 1 & 0,26 \\
\hline Genética (1/2/3) & $2 \% / 3^{\circ} / 6^{\circ}$ & $36 / 36 / 36$ & $36 / 18 / 0$ & $72 / 54 / 36$ & 23 & 5,94 \\
\hline Hematologia (1/2) & $3^{\circ} / 6^{\circ}$ & $36 / 36$ & $18 / 36$ & $54 / 72$ & 19 & 4,91 \\
\hline Anatomia Top./Imagenologia (1/2) & $3 \% / 7^{\circ}$ & $0 / 36 / 36$ & $36 / 0 / 0$ & $36 / 36$ & 31 & 8,01 \\
\hline Imunologia (1-2) & $4^{\circ} / 5^{\circ}$ & $108 / 36$ & $72 / 18$ & $180 / 54$ & 33 & 8,53 \\
\hline IPL/OPL/Gestão de serviços & $2 \% 5^{\circ} / 7^{\circ}$ & $18 / 0 / 36$ & $36 / 36 / 0$ & $54 / 36 / 36$ & 4 & 1,03 \\
\hline Microbiologia (1-2) & $4^{\circ} / 5^{\circ}$ & $108 / 54$ & $72 / 54$ & $180 / 108$ & 55 & 14,21 \\
\hline Parasitologia & $4^{\circ} / 5^{\circ}$ & $108 / 36$ & $72 / 36$ & $180 / 72$ & 35 & 9,04 \\
\hline Processos gerais de patologia & $3^{\circ}$ & 36 & 36 & 72 & 8 & 2,06 \\
\hline Psicologia e Saúde & $2^{\circ}$ & 36 & 0 & 36 & 3 & 0,77 \\
\hline Saúde Coletiva/PIS & $2 \% 3^{\circ} / 4^{\circ}$ & $54 / 54 / 0$ & $0 / 0 / 54$ & $54 / 54 / 54$ & 15 & 3,88 \\
\hline Toxicologia & $6^{\circ}$ & 36 & 18 & 54 & 13 & 3,35 \\
\hline Vigilância sanitária e epidemiológica & $7^{\circ}$ & 36 & 0 & 36 & 15 & 3,88 \\
\hline Virologia & $4^{\circ}$ & 108 & 72 & 180 & 23 & 5,94 \\
\hline Zoologia Médica & $4^{\circ}$ & 36 & 0 & 36 & 9 & 2,33 \\
\hline Sem disciplina relacionada & - & - & - & - & 7 & 1,81 \\
\hline
\end{tabular}

TCC: Trabalho de conclusão de curso. EBMSP: Escola Bahiana de Medicina e Saúde Pública. IPL: Introdução a prática laboratorial. OPL: Oficina de práticas laboratoriais. Anatomia Top: Anatomia topográfica. PIS: Programa interprofissional em saúde. C.H: Carga Horária. Freq: Frequência 


\section{Avaliação temática dos TCCs em relação as áreas da CAPES}

A Tabela 2 exibe a classificação dos TCCs de acordo com a área de conhecimento da CAPES. Foi observado que a maioria dos trabalhos encontram-se na área de Ciências Biológicas [65,12\% (252)], seguido pelas Ciências da Saúde [16,54\% (N=64)], Ciências Humanas [1,03\% (4)], Ciências Exatas e da Terra [0,76\% (3) e Multidisciplinar [1,03\% (4)]. Além destes, grande parte dos trabalhos não corresponderam a nenhuma classificação [15,50\% (60)].

Tabela 2. Classificação dos TCCS de acordo com as áreas do conhecimento da CAPES

\begin{tabular}{|c|c|c|c|c|c|}
\hline $\begin{array}{c}\text { Nível } 1 \\
\text { Grande área }\end{array}$ & $\begin{array}{c}\text { Nível } 2 \\
\text { Área do Conhecimento }\end{array}$ & $\begin{array}{l}\text { Nível } 3 \\
\text { Subárea }\end{array}$ & $\begin{array}{c}\text { Nível } 4 \\
\text { Especialidade }\end{array}$ & $\mathrm{N}$ & $\%$ \\
\hline \multirow{20}{*}{$\begin{array}{l}\text { Ciências } \\
\text { Biológicas } \\
\text { N=252 }\end{array}$} & Ciências Biológicas III & Microbiologia & Bacteriologia & 53 & 13,7 \\
\hline & Ciências Biológicas II & Farmacologia & - & 33 & 8,53 \\
\hline & Ciências Biológicas III & Parasitologia & - & 32 & 8,27 \\
\hline & Ciências Biológicas III & Microbiologia & Virologia & 28 & 7,24 \\
\hline & Ciências Biológicas I & Biologia geral & Genética hum. e med. & 15 & 3,88 \\
\hline & Ciências Biológicas III & Imunologia & Imunologia celular & 14 & 3,62 \\
\hline & Ciências Biológicas III & Imunologia & Imunologia clínica & 14 & 3,62 \\
\hline & Biodiversidade & Zoologia & - & 13 & 3,36 \\
\hline & Ciências Biológicas II & Bioquímica & - & 12 & 3,10 \\
\hline & Ciências Biológicas I & Biologia geral & Genética mol. e microo & 6 & 1,55 \\
\hline & Ciências Biológicas II & Morfologia & - & 6 & 1,55 \\
\hline & Ciências Biológicas II & Morfologia & Citologia e biologia cel & 6 & 1,55 \\
\hline & Ciências Biológicas II & Fisiologia & Neurofisiologia & 4 & 1,03 \\
\hline & Ciências Biológicas II & Farmacologia & Toxicologia & 4 & 1,03 \\
\hline & Ciências Biológicas I & Biologia geral & Mutagênese & 3 & 0,78 \\
\hline & Biodiversidade & Ecologia & Ecologia de ecossist & 3 & 0,78 \\
\hline & Ciências Biológicas III & Imunologia & Imunoquímica & 2 & 0,52 \\
\hline & Ciências Biológicas III & Microbiologia & Micologia & 2 & 0,52 \\
\hline & Ciências Biológicas I & Biologia geral & Genética animal & 1 & 0,26 \\
\hline & Ciências Biológicas I & Biologia geral & Imunogenética & 1 & 0,26 \\
\hline \multirow{8}{*}{$\begin{array}{l}\text { Ciências da } \\
\text { Saúde } \\
\text { N=64 }\end{array}$} & Medicina III & Saúde Coletiva & Saúde Pública & 18 & 4,65 \\
\hline & Medicina III & Saúde Coletiva & Epidemiologia & 11 & 2,84 \\
\hline & Medicina III & Medicina & Ginecologia e obstet. & 10 & 2,58 \\
\hline & Medicina II & Medicina & Hematologia & 10 & 2,58 \\
\hline & Medicina I & Medicina & Clínica Médica & 5 & 1,29 \\
\hline & Farmácia & Farmácia & Farmacognosia & 4 & 1,03 \\
\hline & Medicina II & Medicina & Radiologia médica & 3 & 0,78 \\
\hline & Medicina II & Medicina & Anat. Patol e Patol Clin & 3 & 0,78 \\
\hline \multirow{2}{*}{$\begin{array}{c}\text { Ciências } \\
\text { Humanas } \\
\mathrm{N}=4\end{array}$} & Psicologia & Psicologia & Dist. psicossomaticos & 3 & 0,78 \\
\hline & Educação & Educação & Mét. e Téc. De ensino & 1 & 0,26 \\
\hline $\begin{array}{l}\text { Ciências exatas e } \\
\text { da terra } \quad \mathrm{N}=3\end{array}$ & Astronomia/Física & Física & - & 3 & 0,78 \\
\hline $\begin{array}{l}\text { Multidisciplinar } \\
\qquad N=4\end{array}$ & Biotecnologia & Biotecnologia & - & 4 & 1,03 \\
\hline $\begin{array}{l}\text { Outros } \\
N=60\end{array}$ & - & - & Outros & 60 & 15,50 \\
\hline
\end{tabular}


A figura 8 exibe os dados em relação à classificação da grande área (nível 1) e o total de trabalhos, bem como a estratificação das duas áreas que apresentaram maior número de trabalhos, ciências biológicas e ciências da saúde, respectivamente. Após a estratificação dos dados, observa-se que na área de Ciências Biológicas, a maior parte dos trabalhos foram classificados na área de Ciências Biológicas III [57,54\% (145)]. Já na área de Ciências da Saúde, a maior parte dos trabalhos foram classificados na área de Medicina III [60,94\% (39)].

Figura 8. Dados em relação a classificação das áreas do conhecimento nível 1 e nível 2 x o total de trabalhos
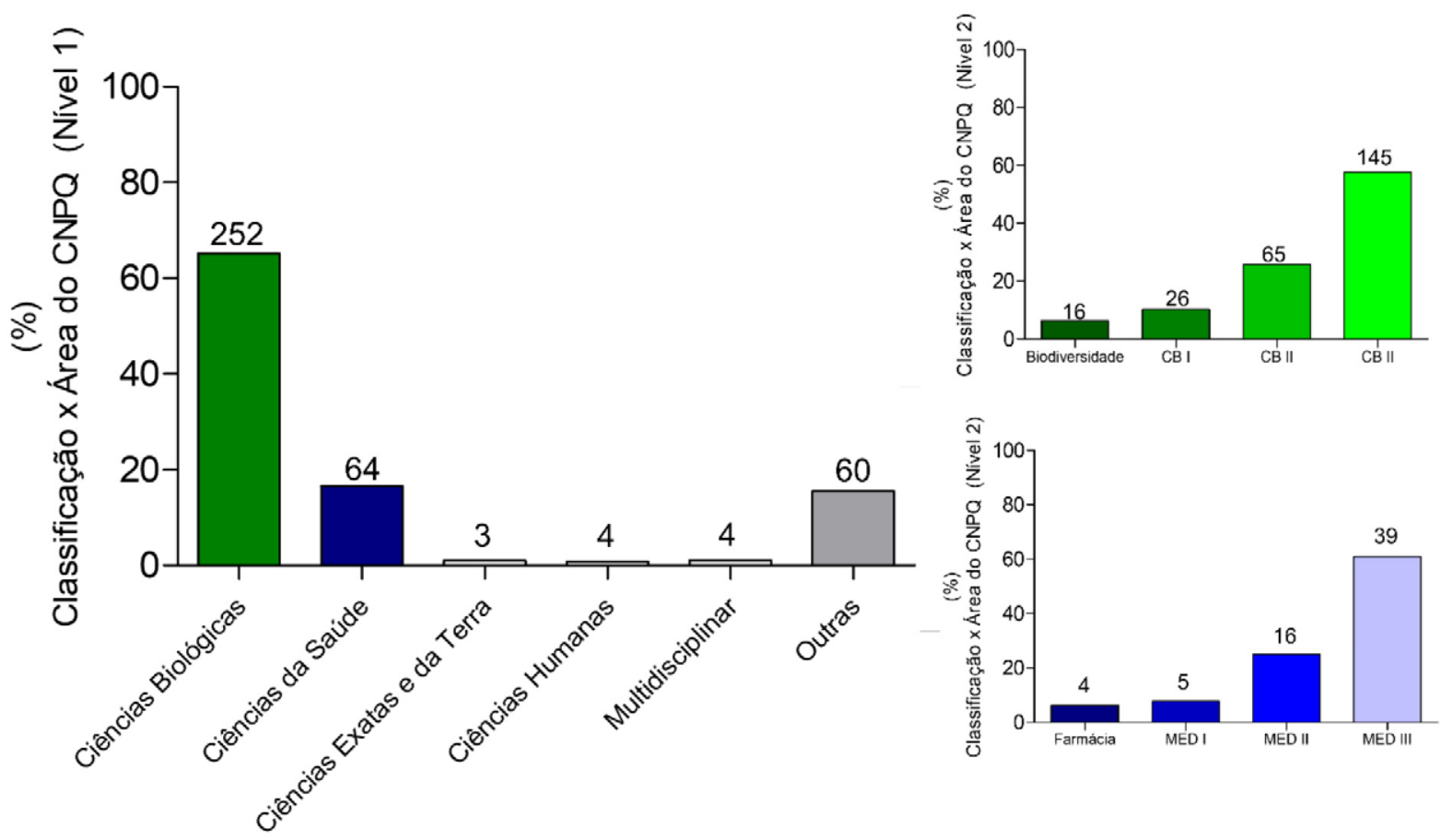

\section{Avaliação temática dos TCCs em relação à habilitação do biomédico}

A disciplina que obteve maior número absoluto de TCCs realizados foi a de Microbiologia, com 55 trabalhos (Figura 7), correspondendo a 14,21\% dos trabalhos, docência e pesquisa [13,95\% (54)]. As áreas de Análises Bromatologicas / Microbiologia de Alimentos e de Biomedicina estética foram as que apresentaram menor frequência de trabalhos relacionados, ambas com 1 trabalho, correspondendo a 0,26\%. 
Figura 7. Representação da avaliação temática dos TCCs em relação a habilitação do biomédico de 2008 a 2018

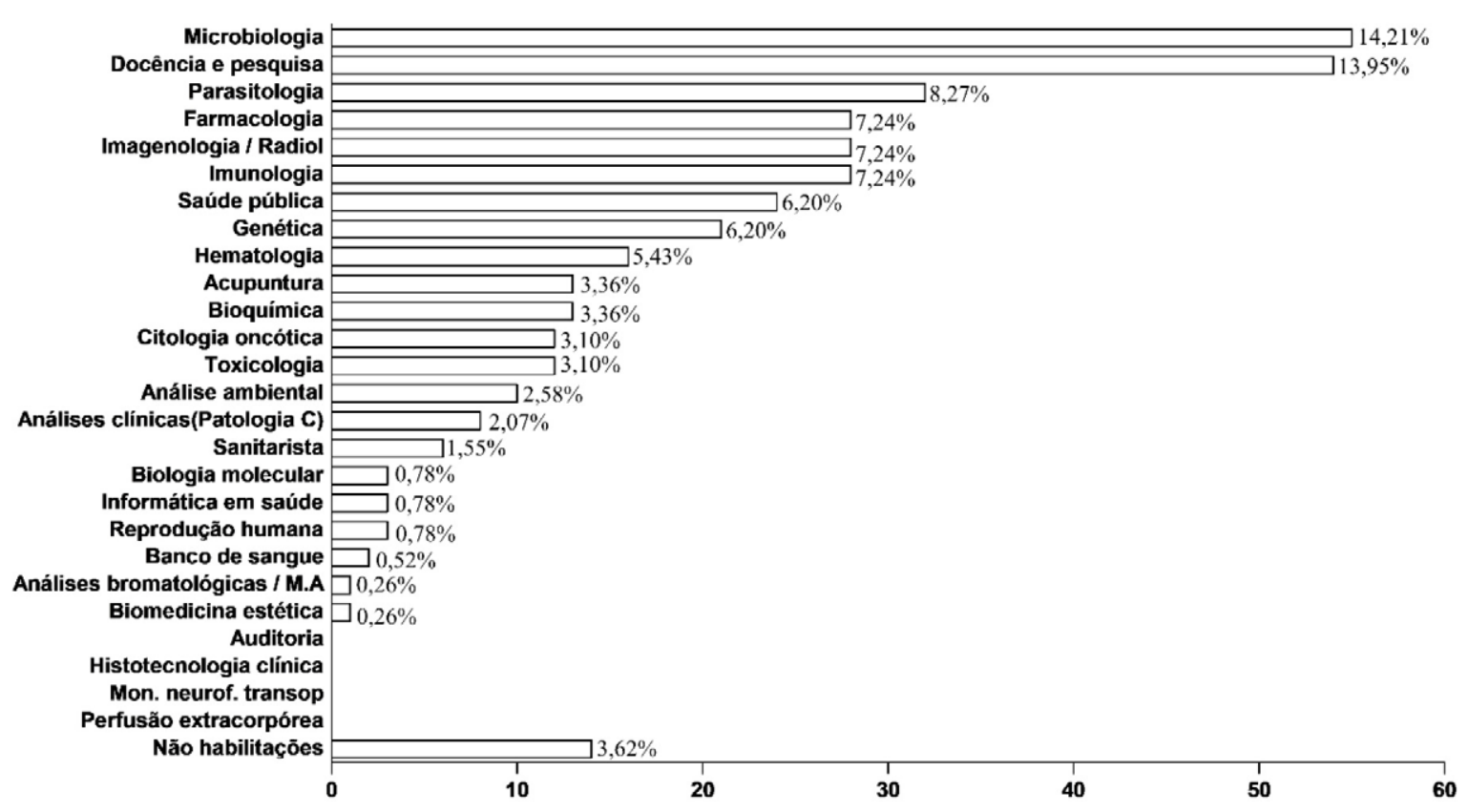

\section{Discussão}

Embora o curso de Biomedicina da EBMSP preconize o trabalho de conclusão de curso como item obrigatório, segundo Costa e cols (2015) esta não é uma exigência do Ministério da Educação ${ }^{10}$.

No presente estudo, observou-se que o número de TCCs recuperados no repositório institucional foi inferior ao número de estudantes formados. Acredita-se que interferências no sistema digital do repositório, como perdas ou dados de arquivos em papel, além de fornecimento errôneo de dados digitais pelos estudantes podem ter ocorrido, bem como a passagem deles para arquivo digital e trabalhos feitos em co-autoria. Nota-se que em 2012 houve poucos trabalhos fornecidos, o que compromete significativamente o número de trabalhos, revisões e experimentais, sendo evidenciados nos gráficos um declínio quando avaliados por ano. Os anos de 2017 e 2018, foram os de menor perda entre o número de formandos e trabalhos encontrados. Segundo Figueiredo \& Drumond (2016), essa disponibilização de TCCs em meio digital ajuda na divulgação desse tipo de criação, e favorece a preservação documental ${ }^{11}$.

Após as análises, foi observado que o número de revisões foi maior que o número de trabalhos experimentais em geral. Apesar disso, os dois primeiros anos avaliados (2008 e 2009), havia um maior número de trabalhos experimentais que revisões, que de- caíram ao longo do tempo. Acredita-se que ao longo dos anos, as novas exigências éticas para trabalhos experimentais foram responsáveis por esta redução, como por exemplo, o processo de submissão ao Comitê de Ética em Pesquisa (CEP). Algumas dificuldades são relatadas pelos pesquisadores para a submissão destes projetos, como a quantidade de documentos, prazos e uma série de dados exigidos pela plataforma Brasil ${ }^{10}$. Apesar da redução ao longo dos anos, nota-se uma recuperação do número de trabalhos experimentais em 2017 e 2018 . Acredita-se que este aumento está relacionado ao aprimoramento dos espaços e da qualidade na pesquisa da Escola Bahiana, com apoio e possibilidades para este tipo de trabalho, como por exemplo, através do Núcleo de Pesquisa e Inovação (NUPI) e iniciações cientificas ${ }^{12}$.

Quando comparado o número de monografias versus o número de artigos científicos, percebe-se que o número de monografias ainda é muito superior. Entretanto, consideramos estes dados enviesados, uma vez que nos períodos de 2006 a 2016.2 não existia a obrigatoriedade pela EBMSP de que os TCCS fossem em formato de artigo científico, então todos foram classificados como sendo uma monografia. Desde 2017.1, os artigos passaram a ser obrigatórios para a conclusão do curso. A exigência da formatação em artigo científico é de grande importância, pois facilita a publicação dos resultados em periódicos científicos, evitando assim que os resultados obtidos permaneçam apenas nas instituições ${ }^{10}$. 
A classificação dos trabalhos em áreas foi de grande dificuldade, devido a temas múltiplos que englobavam diferentes áreas da biomedicina e afins, em um mesmo trabalho. Se fez necessária uma avaliação cautelosa, para garantir uma classificação coerente e equivalente ao trabalho, principalmente na análise das disciplinas da graduação e de áreas da CAPES. Segundo Pedrotti e cols (2016), variadas disciplinas presentes em um curso de graduação são consideradas importantes para a escolha do tema, e para o desenvolvimento do $\mathrm{TCC}^{13}$. Em relação às áreas da CAPES, a maior parte dos trabalhos foi classificado em Ciências Biológicas, provavelmente, devido ao curso de biomedicina estar inserido dentro desta área. O fato do número de TCCs que não correspondem a nenhuma classificação é significativo, possivelmente devido a uma associação com a orientação dos graduandos em relação aos temas e a escolha de acordo com seus estágios e pesquisas.

A disciplina com o maior número de trabalhos foi a Microbiologia, que possui uma carga horária extensa, divididas entres aulas teóricas e práticas, sendo que as práticas servem de atrativo para possíveis TCCs experimentais, o que pode justificar a colocação desta disciplina em primeiro lugar. Em seguida, a Parasitologia, podendo estar associada a grande quantidade de trabalhos e estudos epidemiológicos e experimentais sobre as doenças parasitológicas negligenciadas. Além disso, assim como na Microbiologia, aulas teóricas e práticas, também servem de atrativo para possíveis TCCs. Apesar do estágio I, II e III, também possuir carga horária elevada, não foi muito comtemplado com TCCs, pois a dinâmica operacional acaba não promovendo muitos estudos sobre o estágio e sim sobre setores específicos disponibilizados no local. Talvez, neste caso, inserir com maiores discussões a vigilância sanitária, gestão, auditoria e biotecnologia, podem servir de incentivos aos olhares diferenciados nos estágios, além das habilidades inerentes deste componente curricular. Percebe-se que maiores informações sobre as disciplinas, inclusive aquelas que foram menos contempladas nos TCCs, merecem maiores investigações e contextualizações.

Quando avaliado sobre área de habilitação do biomédico o destaque foi para microbiologia, pois a classificação ocorreu igualmente a disciplina escolhida. Em seguida, a docência e pesquisa foi a segunda área com maior número de trabalhos, esta classificação, só foi possível, uma vez que no dia 01 de maio de 2019, o Conselho Federal de Biomedicina, unificou diversas áreas nesta classificação9. Além disso, a EBMSP, fornece oportunidades do estudante participar de iniciações cientificas. Apesar destes dados, caso fosse somada as habilitações de parasitologia, bioquímica, hematologia, microbiologia e imunologia e as classificasse como análises clínicas, esta seria a habilitação com a maior porcentagem, que até o presente ano é o campo de maior atuação do biomédico.

Este é o primeiro estudo que avalia os trabalhos de conclusão de um curso de graduação em Biomedicina no Brasil. Em outras profissões, outros estudos já realizaram este tipo de avaliação em cursos de graduação, como no curso de odontologia ${ }^{10}$, ciências biológicas $^{13}$, enfermagem ${ }^{3}$, biblioteconomia ${ }^{14}$, arquivologia ${ }^{15}$, design ${ }^{16}$, ciências da informação ${ }^{17}$, dentre outros.

\section{Conclusão}

Conclui-se que o número de TCCs recuperados foi representativo, a maior parte deles foi no formato de revisão de literatura e em estilo de monografia. A disciplina de graduação e área de habilitação do biomédico mais frequente foi a microbiologia. Em relação à área de avaliação da CAPES, Ciências Biológicas foi a mais frequente.

\section{Disponibilidade de dados e materiais}

O conjunto de dados utilizados e/ou analisados no presente estudo estão disponíveis através do autor correspondente.

\section{Contribuições dos autores}

Dias-Lima AG, Queiroz GAN foram responsáveis pela concepção e desenho do estudo. Queiroz GAN, Caldas LSP, Santos YGD foram respónsáveis pela análise dos dados. Queiroz GAN, Caldas LSP, Santos YGD, Dias-Lima AG, Brito VS e Ferraro GA foram responsáveis pela escrita e revisão do artigo. Todos os autores leram e aprovaram a versão final do manuscrito.

\section{Agradecimentos}

Nós agradecemos a Escola Bahiana de Medicina e Saúde Pública e a secretaria acadêmica pelo auxílio na execução e fornecimento de dados para o estudo. 


\section{Conflitos de interesses}

Nenhum conflito financeiro, legal ou político envolvendo terceiros (governo, empresas e fundações privadas, etc.) foi declarado para nenhum aspecto do trabalho submetido (incluindo mas não limitando-se a subvenções e financiamentos, participação em conselho consultivo, desenho de estudo, preparação de manuscrito, análise estatística, etc.).

\section{Referências}

1. Conselho Regional de Biomedicina $2^{\circ}$ Região. Histórico. [Internet]. 2015. Disponível em: http://crbm2.gov.br/website/ historico/

2. Escola Bahiana De Medicina e Saúde Pública; Fundação Bahiana Para o Desenvolvimento Das Ciências. Projeto Político Pedagógico Curso de Biomedicina. [Internet]. 2015. Disponível em: https:// www.bahiana.edu.br/CMS/Uploads/Projeto-Politico-PedagogicoBiomedicina-Bahiana-2015.pdf

3. Car MR, Silva A, Ávila LK. Panorama evolutivo dos trabalhos de conclusão do Curso de Graduação em Enfermagem de 2004 a 2009. Arq Med Hosp Fac Cienc Med Santa Casa São Paulo. 2012; 57(1):30-4.

4. Escola Bahiana de Medicina e Saúde Pública. Trabalhos Científicos. [Internet]. Disponível em: https://www.bahiana.edu. br/especiais/trabalhos-cientificos/

5. Escola Bahiana de Medicina e Saúde Pública. Matriz Curricular Biomedicina. [Internet]. 2017. Disponível em: https://www. bahiana.edu.br/upload/bahiana-matriz-curricular-biomedici na-2017-1-20161122145014.pdf

6. Fundação Coordenação de Aperfeiçoamento de Pessoal de Nível Superior. Tabela Áreas do Conhecimento. [Internet]. 2017. Disponível em: http://www.capes.gov.br/images/documentos/ documentos_diversos_2017/TabelaAreasConhecimento_072012_ atualizada_2017_v2.pdf

7. Fundação Coordenação de Aperfeiçoamento de Pessoal de Nível Superior. Tabelas Áreas do Conhecimento/Avaliação. [Internet]. 2018. Disponível em: https://www.capes.gov. br/avaliacao/instrumentos-de-apoio/tabela-de-areas-doconhecimento-avaliacao

8. Conselho Regional de Biomedicina $5^{\circ}$ Região. Habilitações. [Internet]. Disponível em: http://crbm5.gov.br/site/habilitacoes/

9. Conselho Federal de Biomedicina. Habilitação em “Docência e Pesquisa" unifica especializações da Biomedicina. [Internet]. Disponível em: https://cfbm.gov.br/habilitacao-em-docencia-epesquisa-unifica-especializacoes-da-biomedicina-voltadas-aoensino-e-a-pesquisa/
10. Costa RM, Vieira RS, Cavalcanti NA, Tunes UR, Ribeiro EDP, Oliveira VMB. Perfil da pesquisa acadêmica odontológica da EBMSP. Rev da Abeno. 2015;15(1):70-76. doi: 10.30979/rev.abeno. $\underline{\mathrm{v} 15 i 1.145}$

11. Figueiredo JM, Drumond GM. A Coleção De Tcc's No Repositório Institucional Na Universidade Federal Fluminense: Uma Nova Realidade. XIX Seminário Nacional de Bibliotecas Universitárias. Niterói; 2016.

12. Escola Bahiana de Medicina e Saúde Pública. [Internet]. 2019. [Acesso em 21 de mai. 2019]. Disponível em: https:// www.bahiana.edu.br/pesquisa-e-inovacao/laboratorioscompartilhados/

13. Pedrotti J, Maccali NB, Rempel C, Strohschoen AAG. Temáticas Desenvolvidas em Trabalhos de Conclusão de Curso de Ciências Biológicas. Rev Temp e Esp em Educação. 2016; 9(20):53-62. doi: 10.20952/revtee.v9i20.5893

14. Soares DD. Tendências temáticas e metodológicas das monografias (TCCS) do curso de Biblioteconomia da UFRGS. [TCC]. Porto Alegre: Universidade Federal do Rio Grande do Sul; 2004.

15. Viana GFRV, Madio TCC. Prospecção de Projetos De Pesquisa Em Arquivologia: UFSM. XIV Encontro Nacional de Pesquisa em Ciência da Informação. ENANCIB. Rio Grande do Sul: Universidade Federal de Santa Maria; 2013.

16. Silveira ALM, Ribeiro VGR, Goelzer RT. A produção Monográfica discente da Faculdade de Design do Centro Universitário Ritter dos Reis. 2013: 1-8. doi: 10.13140/RG.2.1.2911.5360

17. Castro Filho CM, Mostafa SP. Panorama Temático de Trabalhos de Conclusão de Curso de Ciências da Informação e da Documentação. XI Encontro Nacional de Pesquisa em Ciência da Informação Inovação e inclusão social: questões contemporâneas da informação. [Internet]. 2010. Disponível em: http://200.20.0.78/ repositorios/bitstream/handle/123456789/1376/Panorama\%20 $-\% 20$ Castro\%20Filho.pdf?sequence $=1$ 